\title{
Impact of Load Variation on Joint Angle Estimation from Surface EMG Signals
}

\author{
Zhichuan Tang, Student Member, IEEE, Hongnian Yu and Shuang Cang
}

\begin{abstract}
Many studies use surface electromyogram (sEMG) signals to estimate the joint angle, for control of upper-limb exoskeletons and prostheses. However, several practical factors still affect its clinical applicability. One of these factors is the load variation during daily use. This paper demonstrates that the load variation can have a substantial impact on performance of elbow angle estimation. This impact leads an increase in mean RMSE (Root-Mean-Square Error) from $7.86^{\circ}$ to $20.44^{\circ}$ in our experimental test. Therefore, we propose three methods to address this issue: 1) pooling the training data from all loads together to form the pooled training data for the training model; 2) adding the measured load value (force sensor) as an additional input; and 3) developing a two-step hybrid estimation approach based on load and sEMG. Experiments are conducted with five subjects to investigate the feasibility of the proposed three methods. The results show that the mean RMSE is reduced from $20.44^{\circ}$ to $13.54^{\circ}$ using method one, $10.47^{\circ}$ using method two, and $8.48^{\circ}$ using method three, respectively. Our study indicates that 1$)$ the proposed methods can improve performance and stability on joint angle estimation and 2) sensor fusion (sEMG sensor and force sensor) is an efficient way to resolve the adverse effect of load variation.
\end{abstract}

Index Terms—sEMG, estimation, sensor fusion, exoskeletons

\section{INTRODUCTION}

S URFACE electromyogram (sEMG) is widely used as the control source for upper-limb exoskeleton and prostheses [1]-[8]. It directly reflects the user's muscle activity level in real time and can be recorded non-invasively from the skin [9]. Control signals to guide the movement of an exoskeleton or prosthetic device, or a virtual environment, can be derived from joint angle estimates obtained from sEMG signals [10]. Kawase et al. [11] used mathematical musculoskeletal models to estimate the joint angles of the arms and hands for the multi-joint exoskeletons (BOTAS) control from EMG signals. Delis et al. [12] proposed a feature extraction and pattern

This work was supported in part by the EU Erasmus Mundus FUSION programme, and by the China Postdoctoral Science Foundation under Project 2015 M581935.

Z. C. Tang is with the Faculty of Science and Technology, Bournemouth University, BH12 5BB Poole, UK and with the College of Computer Science and Technology, Zhejiang University, 310027 Hangzhou, China (e-mail: ttzzcc@zju.edu.cn).

$\mathrm{H}$. $\mathrm{Yu}$ is the corresponding author and with the Faculty of Science and Technology, Bournemouth University, BH12 5BB Poole, UK (e-mail: yuh@bournemouth.ac.uk).

S. Cang is with the Faculty of Management, Bournemouth University, BH12 5BB Poole, UK (e-mail: scang@bournemouth.ac.uk). classification algorithm for estimation of the intended knee joint angle from SEMG signals to control leg prostheses. Aung et al. [13] estimated the shoulder angle based on sEMG signals to control a Virtual Reality (VR) human model under a BPNN controller.

The above work focuses mainly on algorithms to improve the offline estimation performance of pre-recorded signals. These methods may achieve high performance ( $>95 \%$ accuracy) in laboratory conditions [14]. However, applying sEMG to estimate joint angle for the control of exoskeleton and prostheses has several practical limitations [15]. For example, there are more unpredicted variations in a practical use than in laboratory conditions, such as different limb positions [16], signal non-stationarity [8], and electrode shift. Besides these, load variation can significantly affect the joint angle estimation accuracy. For example, in laboratory, the training data can be collected at a predefined load level, and the training strategy of joint angle estimation is able to identify the load level; in practice, an additional load is unpredictable because of different complex upper-limb movements (grasp, seize, lift, etc.), which is in effect increasing the force level required to move the forearm [17]. Researchers have considered the effects of varying load level on the performance of EMG-based pattern recognition systems. It is found that the error rates are between $32 \%$ to $44 \%$ when training and testing data are from different force levels, while the error rates are between $8 \%$ to $19 \%$ when the training and testing data are from the same force level [14]. Changes of the load level may degrade the accuracy of the myoelectric control system by up to $60 \%$ [17]. However, there is little evidence about what will happen to the accuracy of joint angle estimation from sEMG if the load level changes, and there is little research on resolving the effect of load variation on joint angle estimation.

It is difficult to solve this problem using sEMG signals alone, since the load information cannot be acquired from sEMG sensors directly. To address this limitation of multimodal inputs, several studies focused on the sensor fusion technology [18][20]. Fougner et al. [21] used sEMG and accelerometer sensors to solve the adverse effects of limb position on pattern recognition based myoelectric control, which reduces the average classification error from $18 \%$ to $5.7 \%$. Roy et al. [22] developed a combined sEMG and motion sensor system for monitoring activities of daily living in patients with stroke, and could achieve higher classification accuracy using this system. Novak [23] compared different sensors (EMG, EEG, position and so on) to predict targets of human reaching motions, and 
combining different sensors could achieve better prediction performance (up to $90.3 \%$ ). To the best of our knowledge the combination of the sEMG and force sensors has not been used in joint angle estimation.

In this paper, we demonstrate that the load variation can have a substantial impact on the performance of elbow angle estimation, and propose three methods to reduce the effect of load variation:

1) Method One - pooling the training data from all loads together: By pooling the data from different loads in the training phase, we determine if the control system could learn these inter-load differences.

2) Method Two - adding the measured load value as an additional input: This allows the control system to know the loads, which can increase the dimensionality of the estimation algorithm's space. We use a force sensor to measure the load value (the force between palm and dumbbell).

3) Method Three - developing a two-step hybrid estimation approach based on load and sEMG: This method has two steps. In the $1^{\text {st }}$ step, the force data are collected and classified to obtain a specific load; in the $2^{\text {nd }}$ step, the corresponding pre-trained model using the training data from the same single load is applied to estimate the elbow angle.

The rest of this paper is organized as follows. Section II describes the experimental method and the estimation process. Section III presents the results of the experiment and some data analyses. The load effect in elbow angle estimation and the positive effectiveness of estimation performance using three proposed methods are demonstrated using the experimental data. Section IV explains and discusses the experimental results. Section V draws the conclusions.

\section{METHODS}

\section{A. Subjects}

Five male able-bodied subjects (age range: 23-29 years old, height range: $168.4-176.4 \mathrm{~cm}$, weight range: $61-70 \mathrm{~kg}$, all right-handed) participated in this study. The five subjects gave their informed consents prior to the experiments. The experimental protocol was reviewed and approved by the human ethical clearance committee of Zhejiang University. All subjects had a medical examination to eliminate any musculoskeletal and nerve diseases. Before the experiment, they were requested not to participate in any intense upper-limb activities.

\section{B. Experimental Procedure}

Before the experiment, anthropometric measurements (age, height and weight) were recorded and the experimental procedure was explained to the subjects. After the sensors (sEMG sensor, force sensor and motion sensor) were attached on their arms and all signals were normal, subjects wearing a sleeveless shirt were requested to sit and perform elbow flexion-extension movements, as shown in Fig. 1(a). They were asked to maintain shoulder in a constant position at $90^{\circ}\left(\theta_{1}\right)$.
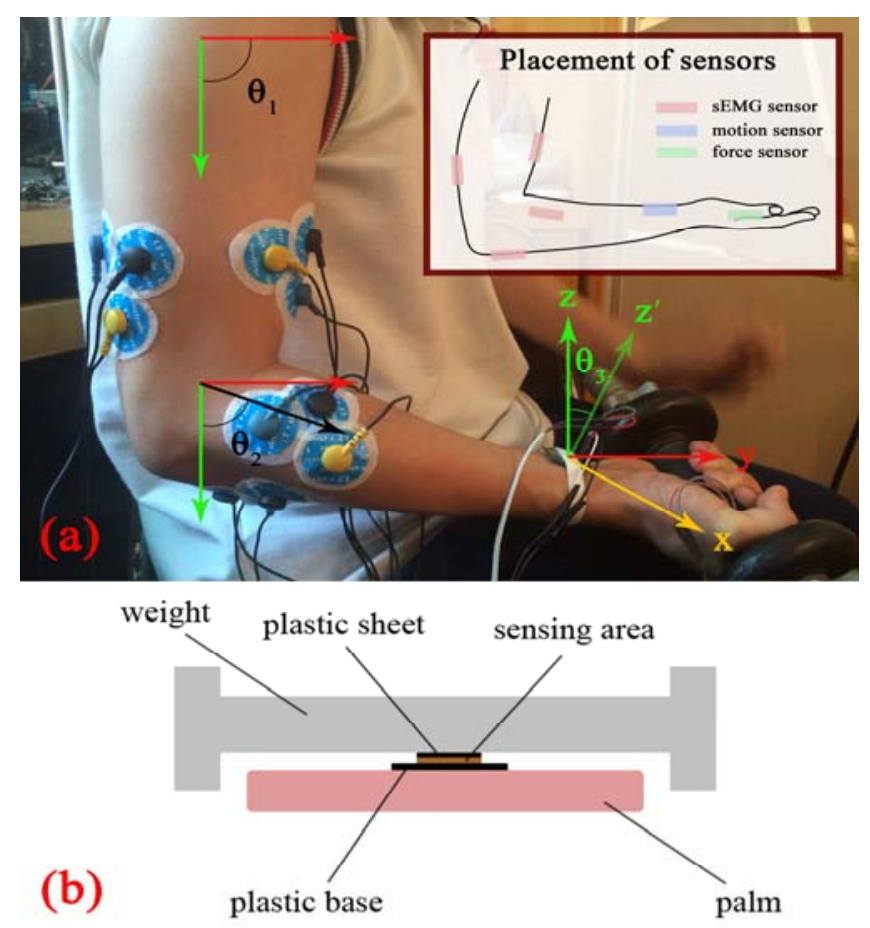

Fig. 1. (a): Experimental setup and the placement of sensors (sEMG sensors, motion sensors and force sensor); (b) the setup of force sensor

TABLE I

THE DIFFERENT CONDITIONS IN NINE TRIALS

\begin{tabular}{|c|c|c|}
\hline Load & Speed & Trials \\
\hline \multirow{3}{*}{ L1: $0 \mathrm{~kg}$} & $\begin{array}{l}\text { V1: constant elbow angular velocity of } \\
90 \% \mathrm{~s}(0.5 \mathrm{~Hz})\end{array}$ & Triall \\
\hline & $\begin{array}{l}\text { V2: constant elbow angular velocity of } \\
45 \% \mathrm{~s}(0.25 \mathrm{~Hz})\end{array}$ & Trial2 \\
\hline & $\begin{array}{l}\text { V3: constant elbow angular velocity of } \\
30 \% \mathrm{~s}(0.17 \mathrm{~Hz})\end{array}$ & Trial3 \\
\hline \multirow{3}{*}{ L2: $1 \mathrm{~kg}$} & $\begin{array}{l}\text { V1: constant elbow angular velocity of } \\
90 \% \mathrm{~s}(0.5 \mathrm{~Hz})\end{array}$ & Trial4 \\
\hline & $\begin{array}{l}\text { V2: constant elbow angular velocity of } \\
45 \% \mathrm{~s}(0.25 \mathrm{~Hz})\end{array}$ & Trial5 \\
\hline & $\begin{array}{l}\text { V3: constant elbow angular velocity of } \\
30 \% \mathrm{~s}(0.17 \mathrm{~Hz})\end{array}$ & Trial6 \\
\hline \multirow{3}{*}{ L3: $2 \mathrm{~kg}$} & $\begin{array}{l}\text { V1: constant elbow angular velocity of } \\
90 \% \mathrm{~s}(0.5 \mathrm{~Hz})\end{array}$ & Trial7 \\
\hline & $\begin{array}{l}\text { V2: constant elbow angular velocity of } \\
45^{\circ} / \mathrm{s}(0.25 \mathrm{~Hz})\end{array}$ & Trial8 \\
\hline & $\begin{array}{l}\text { V3: constant elbow angular velocity of } \\
30 \% \mathrm{~s}(0.17 \mathrm{~Hz})\end{array}$ & Trial9 \\
\hline
\end{tabular}

$\mathrm{kg}=$ kilogram, $\mathrm{s}=$ second, $\mathrm{Hz}=$ hertz.

The angle range of the elbow $\left(\theta_{2}\right)$ was from $0^{\circ}$ (full extension) to $90^{\circ}$ (flexion). The palm was supinated and kept horizontal through all trials shown in Fig. 1(a), and the motion was limited to the sagittal plane.

The subjects were instructed to perform the elbow flexion-extension movements under three different loads: $0 \mathrm{~kg}$ (no load), $1 \mathrm{~kg}$, and $2 \mathrm{~kg}$, respectively. A dumbbell with different weight plates was used in load variation. For each of three loads, subjects performed fifty repetitions continuously at three speeds (as shown in Table I, called a trial). This resulted in a 
total data set of 5 subjects $\times 50$ repetitions $\times 3$ loads $\times 3$ speeds. A metronome was used to guide the flexion-extension movements at different speeds [24]. The subjects finished an elbow flexion and elbow extension within the time interval of two metronome beeps. The subjects were instructed to move smoothly across the range and not to delay at either end.

Consecutive trials were separated by resting periods of 4-6 minutes to avoid fatigue. To avoid fatigue during each trial, subjects were asked to rest if they felt fatigue or could no longer perform the repetitions. At the same time, the experimenter was observing the change of median frequency (MF) of four muscles in real time. A shift of MF to the low end indicated muscle fatigue [25]. These two tests ensured that all records were fatigue free. The average duration of the experiment was approximately 80 minutes for each subject.

\section{Data Acquisition}

The movement of the elbow joint in this experiment included two parts: flexion and extension. The agonistic muscle and the synergistic muscle of the flexion movement are the biceps brachii and the brachioradialis. The agonistic muscle and the synergistic muscle of the extension movement are the triceps brachii and the anconeus [26]. The four sEMG sensors (MyoScan, Thought Technology Ltd., Canada) were used to collect sEMG signals of the four muscles in the experiment. The parameters of the sensors are: range of $0-2000 \mu \mathrm{V}$, input impedance $>10 \mathrm{G} \Omega$ in parallel with $10 \mathrm{pF}, \mathrm{CMRR}>130 \mathrm{~dB}$ and input/output gain=500. Before electrode attachment, alcohol was used to clean the skin, and conductive gel was used to improve the contact of the electrode with the skin [27]. Then, the pairs of triode electrodes of the sEMG sensors were attached to the four muscles of the subject's right arm. The inter-electrode distance was $2 \mathrm{~cm}$. The placement of electrodes was in the direction of the muscle fibers on the midline of the muscle belly to avoid the innervation zone of the muscles [28]. sEMG signals were amplified by a factor of 2000 and sampled at $1024 \mathrm{~Hz}$

One force sensor (FlexiForce, Tekscan Inc., USA; force range: $0-111 \mathrm{~N}$ ) was used to measure the load variation. As shown in Fig. 1(b), there is a hard plastic base placed on the bottom of the sensing area; a hard plastic sheet, covering only the sensing area, is placed on top of the sensing area. Plastic base, sensing area and plastic sheet are fixed together using glue. Then these are fixed between the palm and weight using tape. When there was a force (dumbbell) on the sensing area of the force sensor, the corresponding output voltage was calculated as

$$
V_{\text {OUT }}=-V_{T} \times\left(R_{F} / R_{S}\right)
$$

where $V_{T}$ is the drive voltage, $R_{F}$ is the reference resistance and $R_{S}$ is the sensor resistance. Then the output voltage is converted to the corresponding force data using a 12-bit A/D converter.

One 6-axis motion sensor (MPU6050, InvenSense Inc., California: 3 -axis gyroscope +3 -axis accelerometer) was used to acquire the data of elbow angle. The parameters of the sensor are: angular velocity range: $\pm 2000 \%$, and acceleration range: $\pm 16 \mathrm{~g}$. The sensor was placed about $30 \mathrm{~mm}$ from the palm on the midline of forearm. The 16-bit A/D converter module on the motion sensor gave the angular velocity data directly. The angular acceleration can be calculated by

$$
a=\lim _{\Delta t \rightarrow 0} \frac{\Delta \omega}{\Delta t}
$$

where $\Delta t$ is the time interval, and $\Delta \omega$ is the change of the angular velocity over the time interval. The elbow angle $\theta_{2}$ is

$$
\theta_{2}=90^{\circ}-\theta_{3}=90^{\circ}-\tan ^{-1}\left(\frac{\sqrt{a_{x}^{2}+a_{y}^{2}}}{a_{z}}\right)
$$

where $\theta_{3}$ is the angle between $\mathrm{x}$-axis of natural coordinates and $\mathrm{x}$-axis of the motion sensor, $a_{x}$ is x-axis angle acceleration, $a_{y}$ is $\mathrm{y}$-axis angle acceleration and $a_{z}$ is z-axis angle acceleration. The range of $\theta_{2}$ and $\theta_{3}$ is $\pm 90^{\circ}$.

All force data and angle data were sampled at $1024 \mathrm{~Hz}$ and synchronized with the corresponding EMG signal.

\section{Data Processing}

The sEMG, force and angle data were processed offline. The sEMG signal was band-pass filtered at $10-400 \mathrm{~Hz}$ with a notch filter implemented to remove the $50 \mathrm{~Hz}$ line interference. The force and angle data were low-pass filtered at $6 \mathrm{~Hz}$.

All data were segmented for feature extraction using the overlapped windowing technique [29]. The analysis windows had duration of $70 \mathrm{~ms}$ for feature extraction and were overlapped by $20 \mathrm{~ms}$. The RMS (root mean square), as one of the simple time domain features from sEMG signals is widely accepted [30]-[32]. It was therefore adopted in this study as below

$$
R M S=\sqrt{\frac{1}{N} \sum_{i=1}^{N} v_{i}^{2}}
$$

where $v_{i}$ is the voltage at the $i$ th sampling and $N$ is the number of sampling points. In each analysis window, the mean values of the force and angle data are

$$
\begin{aligned}
& \bar{F}=\frac{1}{N} \sum_{i=1}^{N} F_{i} \\
& \bar{A}=\frac{1}{N} \sum_{i=1}^{N} A_{i}
\end{aligned}
$$

where $\bar{F}$ is the mean force, $\bar{A}$ is the mean angle, $F_{i}$ is the force at the $i$ th sampling, $A_{i}$ is the angle at the $i$ th sampling and $N$ is the number of sampling points.

Back-propagation neural networks (BPNN) were used to learn the association between the sEMG and elbow angle. The inputs of the BPNNs are sEMG features (there was an additional force input in Method Two) using the 70-ms long analysis window. The outputs of BPNNs are the respective mean angle of the corresponding analysis windows. Network structure of neural network, like the number of nodes in the hidden layer, was not the focus of this work, but this might affect the estimation performance. To select the best network structure, we set different numbers of nodes (from 2 to 20) in hidden layer to adjust the network structure and compared the 


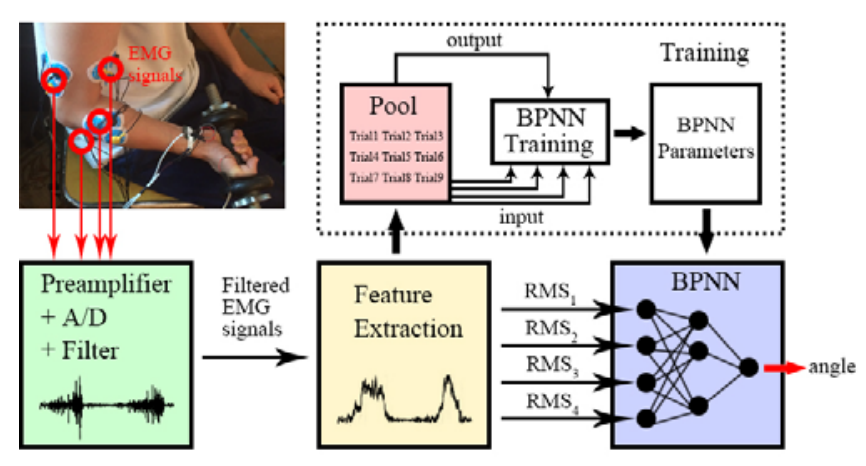

Fig. 2. The estimation scheme of Method One: After the preprocessing, extract the features from the filtered sEMG signals. Under each speed, pool all the training data together to form the pooled training data for the BPNN.
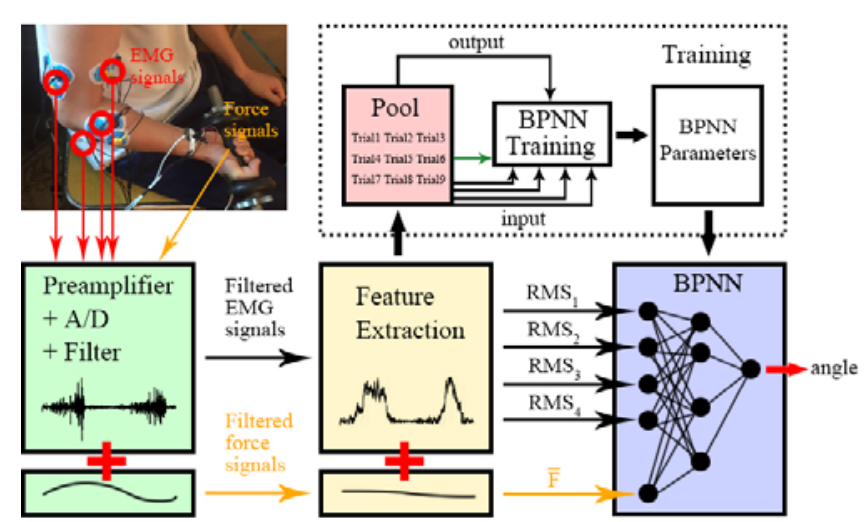

Fig. 3. The estimation scheme of Method Two: After the preprocessing, extract the features from the filtered sEMG signals and force signals (additional input). Under each speed, pool all the training data together to form the pooled training data for the BPNN.

results. A sigmoid function was used for the transfer function when training the network. The output $y$ is

$$
y=f\left(\sum w_{i} x_{i}\right)=\frac{1}{1+e^{-\left(\sum w_{i} x_{i}\right)}}
$$

where $x_{i}$ is the input, $w_{i}$ is the weighting factor attached to that input, $e$ is the exponential function and $f()$ is the transfer function. When the training finished, the model of sEMG-Angle was built, namely the mapping from four muscles' sEMG signals to elbow angle was found.

A support vector machine (SVM) classifier was used to recognize a specific load in Method Three because of its remarkable robustness and high performance across a wide variety of applications [33]. The force data were used for load classification $(0 \mathrm{~kg}, 1 \mathrm{~kg}$ and $2 \mathrm{~kg})$. LIBSVM library was employed as the core of the SVM classifier [34].

\section{E. Estimation}

To investigate the effect of load variation on elbow joint angle estimation, the BPNNs were trained using data from each load (three models in each speed and totally nine trials) and

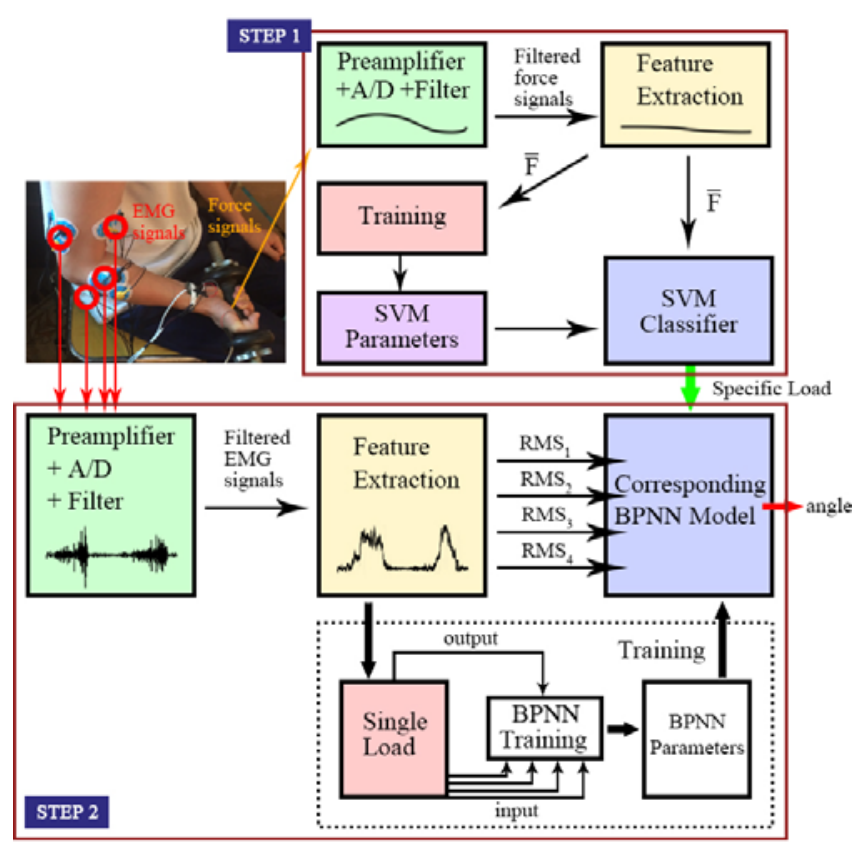

Fig. 4. The estimation scheme of Method Three: In the first step, collect force data and classify them to recognize a specific load; in the second step, apply the corresponding pre-trained model using training data from the same single load to estimate the elbow angle.

tested in all loads under each speed. The inputs of BPNNs are four muscles' RMS, and the desired output is the elbow angle. For each trial, the randomly selected $80 \%$ of all data was used as the training data in train phase. The remaining $20 \%$ data was used as the testing data. In train phase, we used 10-fold cross-validation procedure (where 9 folds were used for training and 1 fold was used for validation) on the corresponding training data ( $80 \%$ of all data). Under each speed, we evaluated the estimation performance of intra-load when the training and testing data were from the same load and that of inter-load when the training and testing data were from different loads.

In order to resolve the effect of load variation, we propose the following three methods:

1) Method One: pooling training data from all loads together By pooling data from different loads in the training phase, we can determine if the control system could learn these inter-load differences. The inputs of BPNNs are four muscles' RMS, and the desired output is the elbow angle. Under each speed, the training data from all three loads (randomly selected $80 \%$ data in each load) are used together to form the pooled training data for the BPNN. The testing data from all three loads (the remaining 20\% data in each load) are used together to form the pooled testing data. 10 -fold cross-validation is applied in train phase. The estimation scheme is shown in Fig. 2.

2) Method Two: adding the measured load value as an additional input 
(a)
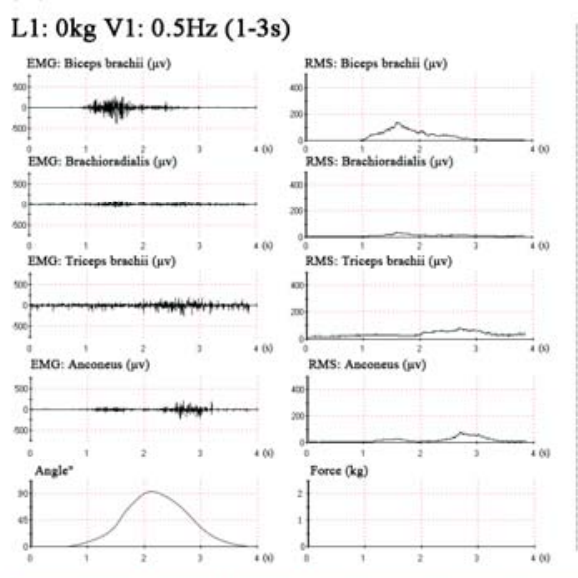

L2: $1 \mathrm{~kg} \mathrm{V1:} 0.5 \mathrm{~Hz}(1-3 \mathrm{~s})$

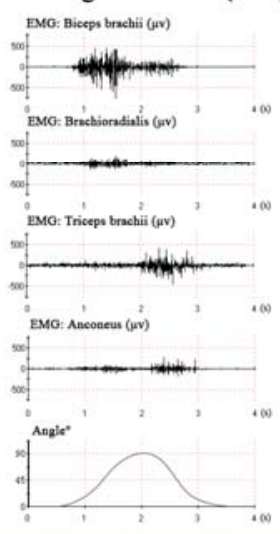

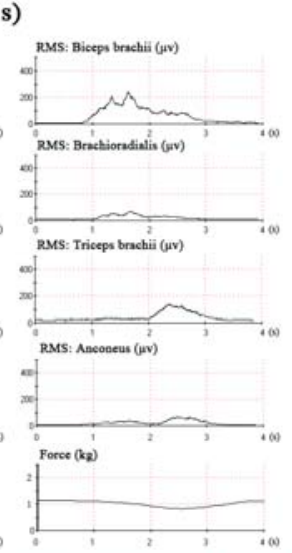

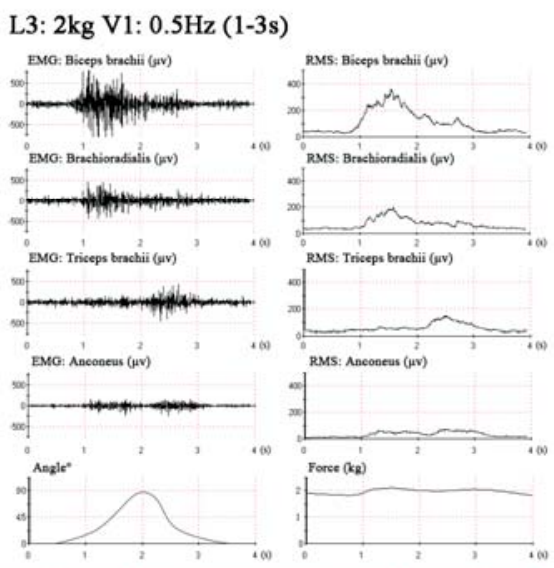

(b)

V1: $0.5 \mathrm{~Hz}(1-3 \mathrm{~s}) \mathrm{L} 2: 1 \mathrm{~kg}$

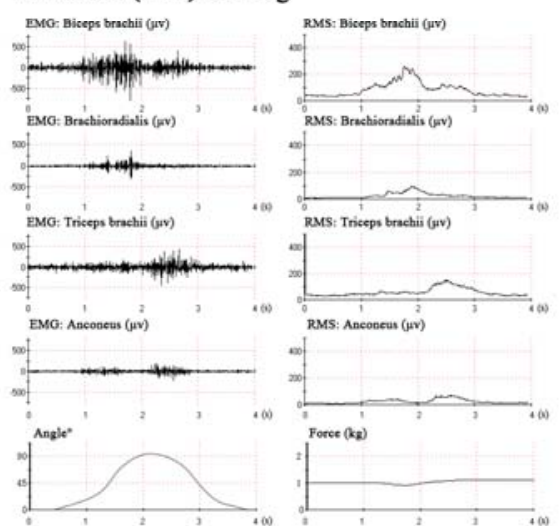

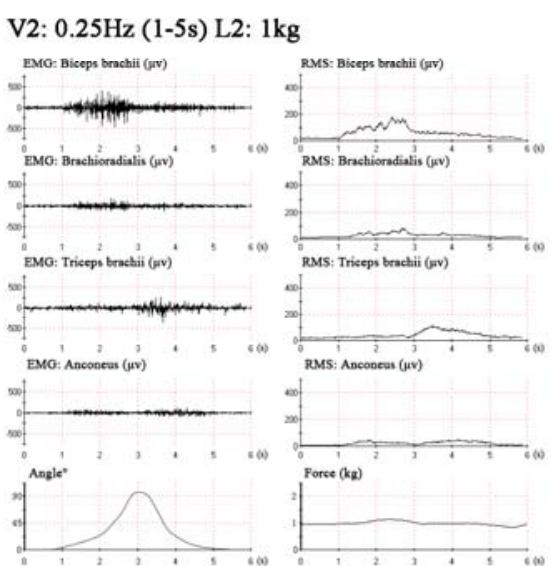

V3: $0.17 \mathrm{~Hz}(1-7 \mathrm{~s}) \mathrm{L} 2: 1 \mathrm{~kg}$

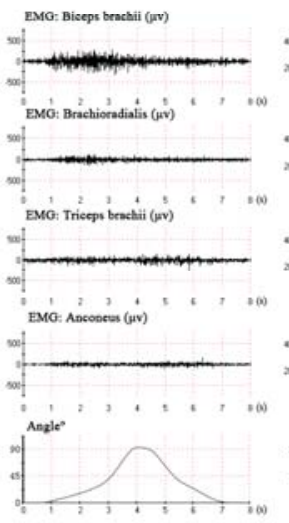

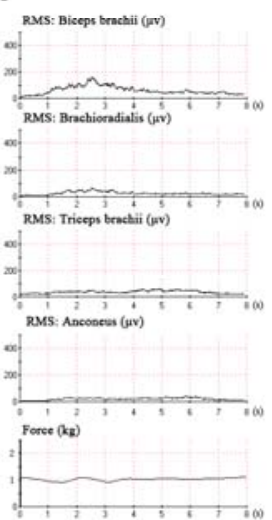

Fig. 5. The data (sEMG, RMS, actual angle and force data) of flexion-extension movements. (a): a representative example of one subject at V1 and three loads; (b) a representative example of one subject at L2 and three speeds.

Testing loads

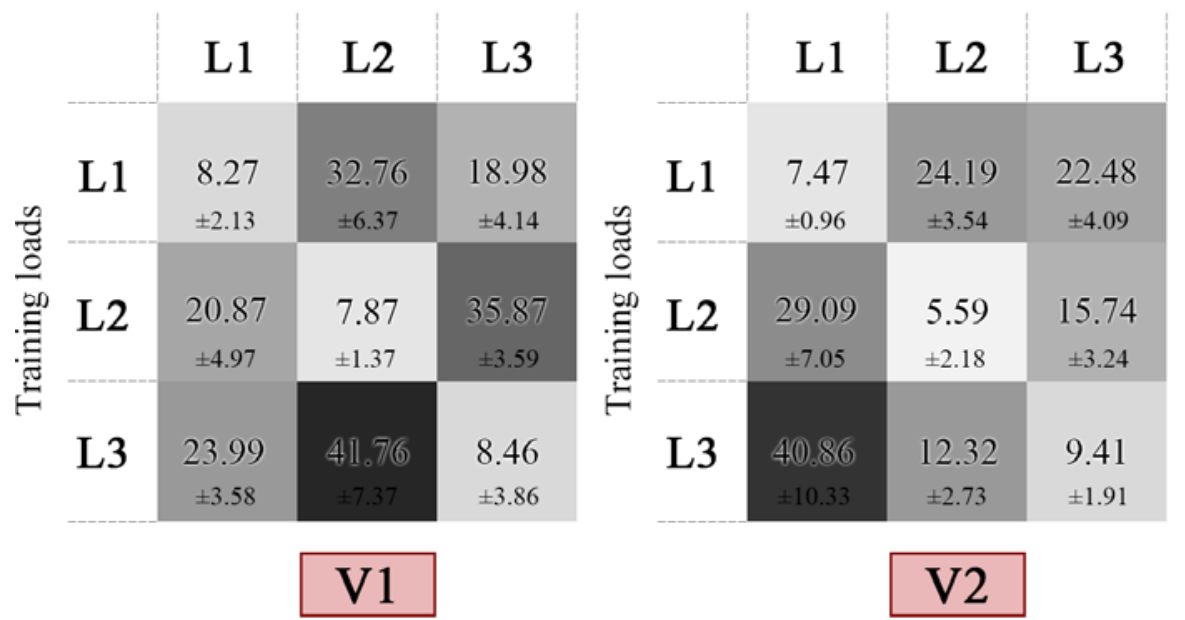

Testing loads

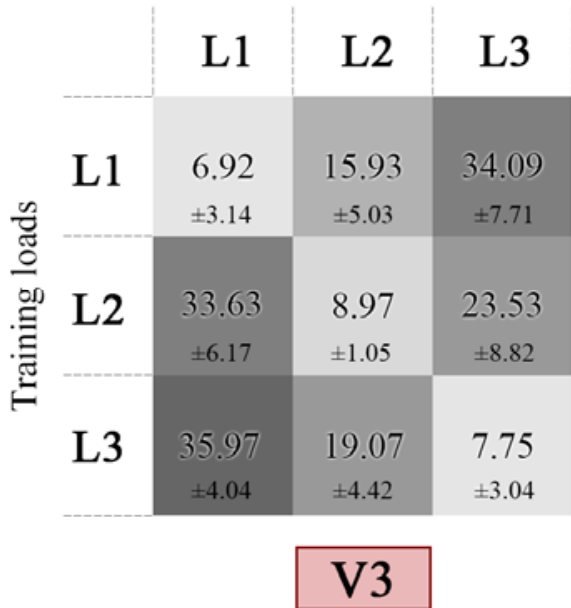

Fig. 6. RMSE (mean $\pm \mathrm{sd}$ ) resulting matrices under three speeds. Each entry in the matrix represents the RMSE (mean \pm sd) across all subjects for the indicated training and testing loads (vertical and horizontal axis, respectively). Darker color indicates larger RMSE.

Measure the load value by a force sensor which is used as an additional input for the BPNNs. This increases the dimensionality of the estimation algorithm's space. The inputs of BPNNs are four muscles' RMS and the force data, forming the feature vectors: 
TABLE II

THE INTRA-LOAD RMSES (MEAN \pm SD), THE INTER-LOAD RMSEs (MEAN \pm SD) AND THE OVERALL RMSEs (MEAN \pm SD) Of THREE RESULTING MATRICES

\begin{tabular}{ccccc}
\hline \hline & V1 & V2 & V3 & Total \\
\hline intra-load RMSEs (mean \pm sd) & $8.20 \pm 0.30$ & $7.49 \pm 1.91$ & $7.88 \pm 1.03$ & $7.86 \pm 1.14$ \\
inter-load RMSEs (mean \pm sd) & $29.04 \pm 9.12$ & $24.11 \pm 10.17$ & $27.03 \pm 8.63$ & $26.73 \pm 9.01$ \\
overall RMSEs (mean \pm sd) & $22.09 \pm 12.67$ & $18.57 \pm 11.60$ & $20.65 \pm 11.77$ & $20.44 \pm 11.65$ \\
\hline \hline
\end{tabular}

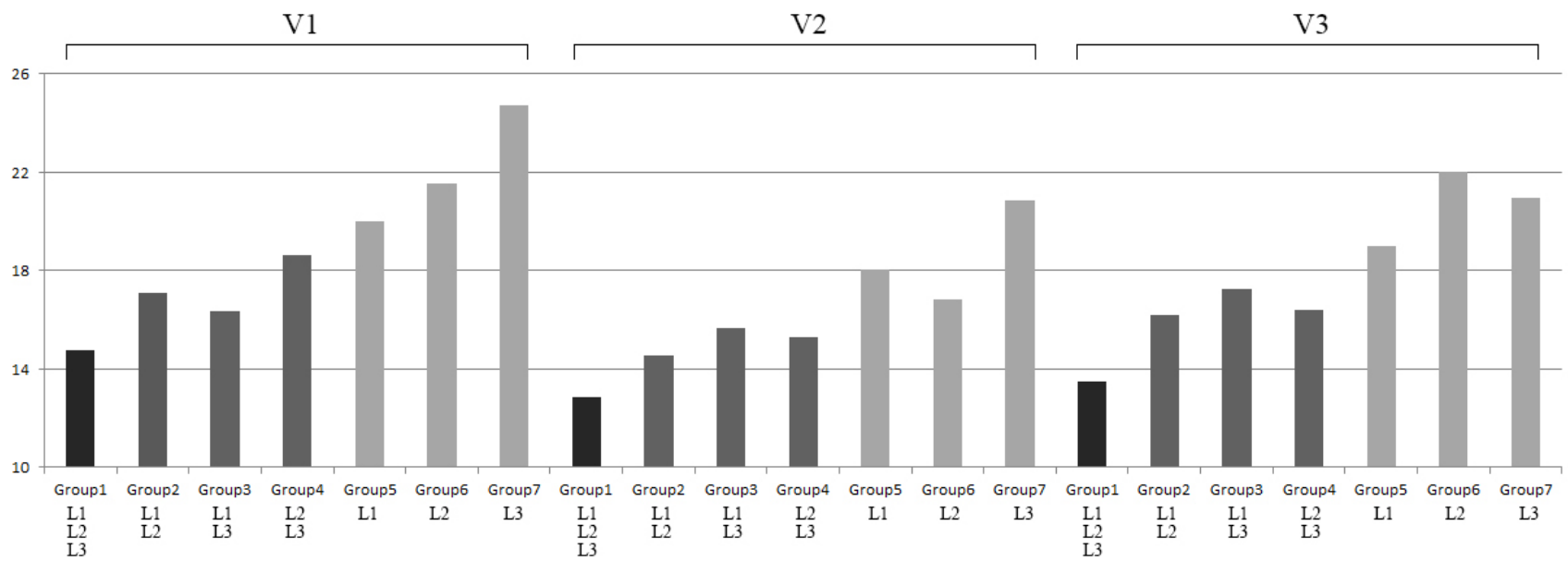

Fig. 7. The mean RMSEs of all groups under each speed across all subjects. For each group, the training data were from one load, two loads or three loads, and the testing data were from all three loads.

$$
\left[\left(R M S_{i}\right)_{n}, \bar{F}_{n}\right]
$$

where $i$ is electrode number and $n$ is sampling points. And the desired output is the elbow angle. Under each speed, the training data from all three loads (randomly selected $80 \%$ data in each load) are used together to form the pooled training data for the BPNN. The testing data from all three loads (the remaining $20 \%$ data in each load) are used together to form the pooled testing data. 10-fold cross-validation is applied in train phase. The estimation scheme is shown in Fig. 3.

3) Method Three: developing a two-step hybrid estimation approach based on load and sEMG

Under each speed, in the first step, classify the force data to recognize a specific load $(0 \mathrm{~kg}, 1 \mathrm{~kg}$ and $2 \mathrm{~kg})$ using a $\mathrm{SVM}$ classifier. For all nine trials, use the training data from all three loads (randomly selected $80 \%$ data in each load) together to form the pooled training data for the SVM. Use the testing data from all three loads (the remaining $20 \%$ data in each load) together to form the pooled testing data. 10 -fold cross-validation is applied in train phase. After training, we could obtain a specific load according to the force data. In the second step, apply the corresponding pre-trained model using training data from the same single load to estimate the elbow angle. The estimation scheme is shown in Fig. 4.

The RMSE (root-mean-square error) is used to evaluate estimation performance of BPNNs. The RMSE between the actual angle and the estimated angle is

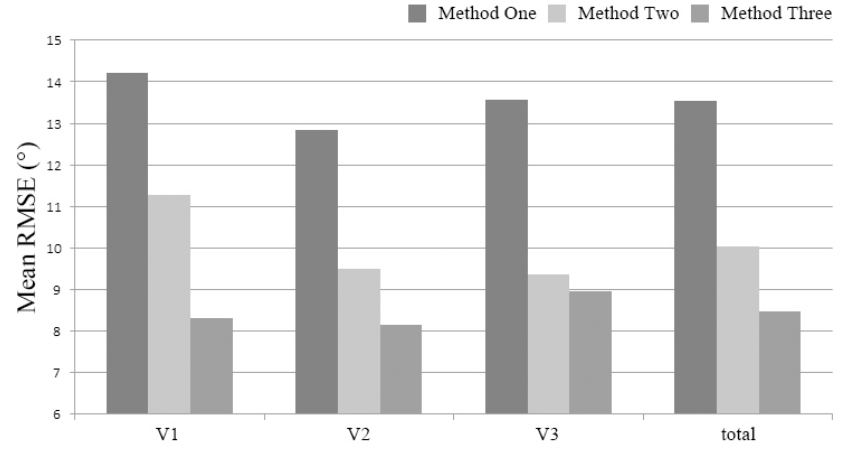

Fig. 8. The mean RMSE of three methods under three speeds across all subjects.

$$
R M S E=\sqrt{\frac{1}{N} \sum_{i=1}^{N}\left(A_{i}-A_{i}^{\prime}\right)^{2}}
$$

where $A_{i}$ is the actual angle, $A_{i}^{\prime}$ is the predicted angle and $N$ is the number of testing data.

\section{RESULTS}

All data were collected and processed from the five subjects in the experiment. The data (sEMG, RMS, actual angle and force data) of flexion-extension movements are shown in Fig. 5(a) (a representative example of one subject at V1 and three loads) and Fig. 5(b) (a representative example of one subject at L2 and three speeds). To compare different numbers of nodes in 


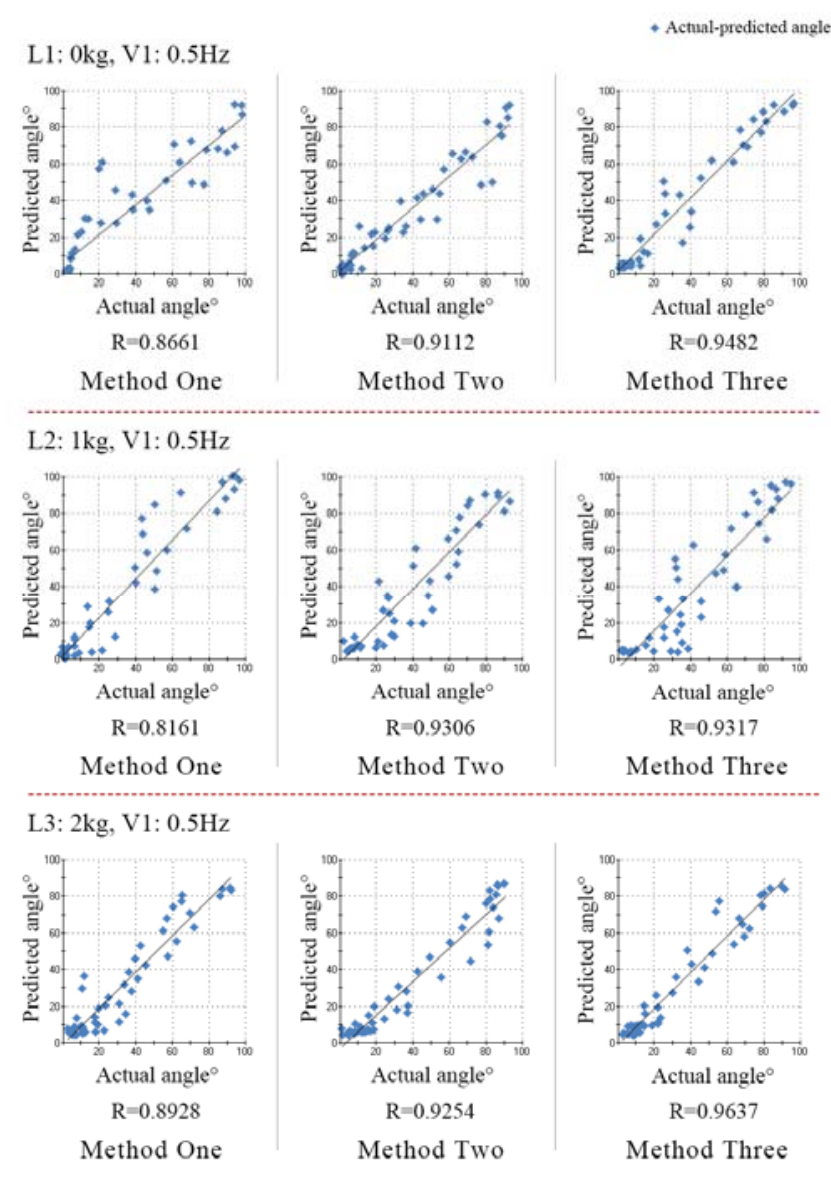

Fig. 9. The correlation diagram and correlation coefficient between the actual angle and the predicted angle of three methods (the testing data are from one repetition of one subject at V1 and three loads).

hidden layer (from 2 to 20), for each one, mean RMSEs of the cases where the training data and testing data are from the same load under three speeds are calculated. The maximum mean RMSE is $8.83^{\circ}$ when number of nodes is 2 , the minimum mean RMSE is $7.71^{\circ}$ when number of nodes is 16 , and the overall mean RMSE is $8.19^{\circ}$. Through t-test (data are normal distribution by using Shapiro-Wilk test), there is no significant difference $(p>0.05)$ between the RMSEs of 3 nodes (mean RMSE is $7.86^{\circ}$ ) and the RMSEs of 16 nodes (mean RMSE is $7.71^{\circ}$ ) across all subjects. We set 3 nodes in hidden layer for simplifying model structure and improving learning speed [35].

\section{A. Effect of Load Variation on Elbow Joint Estimation}

Under each speed, three different BPNN models were trained; each one used data from one load and was tested in all loads. The resulting matrices are shown in Fig. 6. Each entry in the matrix represents the RMSE (mean $\pm \mathrm{sd}$ ) across all subjects for the indicated training and testing loads (vertical and horizontal axis, respectively). The RMSEs shown in the main diagonal are for the cases where the training data and the testing data are from the same load (intra-load), and in the off-diagonal are for the cases where the training data and the testing data are from the different loads (inter-load). The intra-load RMSEs in main diagonal and the inter-load RMSEs in off-diagonal are significantly different $(p<0.005)$ according to the $t$-test. This implies that the load variation has a substantial impact on the performance of elbow angle estimation. For example, the results of training with L3 and testing with L2 or vice versa $\left(41.76^{\circ}\right.$ and $35.87^{\circ}$, respectively) under $\mathrm{V} 1$ are poorest; similarly, the results for L1-L2 (V1), L3-L1 (V2), L3-L1 (V3) and L1-L3 (V3) $\left(32.76^{\circ}, 40.86^{\circ}, 35.97^{\circ}\right.$ and $34.09^{\circ}$, respectively) are poor as well, but better than those of L3-L2 and L2-L3.

The intra-load RMSEs (mean $\pm \mathrm{sd}$ ), the inter-load RMSEs (mean $\pm \mathrm{sd}$ ) and the overall RMSEs (mean \pm sd) of three resulting matrices are shown in Table II. The total mean intra-load RMSE is $7.86^{\circ}$, whereas the total mean inter-load RMSE and total mean overall RMSE are $26.73^{\circ}$ and $20.44^{\circ}$, respectively. We also find that different speeds affect the performance of elbow joint estimation in Fig. 5(b) and Table II. For example the total mean overall RMSE under V2 $\left(18.57^{\circ}\right)$ is smaller than those under V1 $\left(22.09^{\circ}\right)$ and V3 $\left(20.65^{\circ}\right)$.

\section{B. Results of Three Methods}

In Method One, we pooled the training data from all loads together. To investigate the effect of pooling, we constructed different training set combinations into seven groups for comparison. For each group, the training data were from one load, two loads or three loads, and the testing data were from all three loads. The mean RMSEs of all groups under each speed are shown in Fig. 7. We find that the mean RMSE increases gradually from Group1 (training with L1, L2 and L3) to Group7 (training with L3) under each speed. The results imply that when one training set combination included more loads, the estimation performance would be better. The estimation performance of the BPNN model using training data from only one load is poorer than that of the others.

In Method Two, the load value measured by a force sensor was used as an additional input for the BPNNs. In Method Three, under each speed, the force data were used for load classification. There was a zero load classification error using a SVM classifier to get a specific load. The corresponding pre-trained model using the training data from the same single load was applied to estimate the elbow angle. The mean RMSEs of three methods under three speeds are shown in Fig. 8. It is found that Method Three provides the best estimation performance among three methods for all three speeds and total mean RMSE $\left(8.31^{\circ}, 8.16^{\circ}, 8.97^{\circ}\right.$ and $8.48^{\circ}$, respectively). However, RMSEs of all three methods are smaller than that of the conventional method $\left(20.44^{\circ}\right)$ trained using data from single load and tested in all loads.

For a further comparison of the three methods, correlation coefficient $(R)$ between the actual angle and the predicted angle is used to assess the estimation performance. When the value of $R$ is closer to 1 , there is a smaller error between the actual angle and the predicted angle. The correlation diagram and correlation coefficient between the actual angle and the predicted angle of three methods (the testing data are from one repetition of one subject at V1 and three loads) are shown in Fig. 
9. There is a smallest error between the actual angle and the predicted angle using Method Three at V1 and three loads $(R=0.9482,0.9317$ and 0.9637 . respectively).

\section{DISCUSSION}

Table II shows that a total mean intra-load RMSE $\left(7.86^{\circ}\right)$ when the training data and testing data are from the same load is significantly smaller than the corresponding total mean inter-load RMSE $\left(26.73^{\circ}\right)$ when the training data and testing data are from the different loads. The results indicate that elbow angle estimation using sEMG strongly depends on load variation. Different load levels produce different muscle activity levels, and sEMG signals directly reflect the muscle activity level in real time [1]. On the other hand, there are several other effects such as variations in muscle recruitment (due to different muscle forces) and the force-length relationship of the muscle [21]. As a result, training an exoskeleton (or prosthesis) control system in a single load may be insufficient if the system is required to perform well in multi-load applications. The degradation due to load variation may be significantly different between laboratory and practice. The previous studies discussed the load effect in the performance of pattern recognition. Al-Timemy [17] focused on investigating the effect of the change in force levels on the performance of pattern recognition systems. Results showed that changing the force level degraded the performance of the myoelectric control system by up to $60 \%$. The Scheme and Englehart [14] conducted an experiment in which users performed contractions at 20 to 80 percent of the strongest contraction to demonstrate the impact of the force variation. They trained a classifier using data from each force level and tested it at each level, and the average error was up to $45 \%$. However, few studies focus on the effect of load variation on joint angle estimation and how to resolve it like our present study.

We proposed three methods to solve this problem in this paper. Method One reduces the total mean overall RMSE from $20.44^{\circ}$ to $13.54^{\circ}$ by pooling training data from all loads together. Since training in multiple loads is time consuming, it is desirable to reduce the number of training loads. However, the mean RMSE increases as fewer loads (from Group1 to Group7) are included in the training set under each speed. The results imply that when one training set combination included more loads, the estimation performance would be better. The estimation performance of the BPNN model using training data from only one load is poorer. Method Two reduces the total mean overall RMSE from $20.44^{\circ}$ to $10.05^{\circ}$ by using the load value measured by a force sensor as an additional input for the BPNNs, as shown in Fig. 8. In the first step of Method Three, the force sensor data are used for the loads classification, which is able to identify a specific load accurately. Then, the corresponding pre-trained model using the training data from the same single load is applied to estimate the elbow angle, and the total mean overall RMSE further reduces to $8.48^{\circ}$. We have shown that the estimation performance of Method Three is better than that of Method Two. One possible reason is that, although Method Two using the additional input (force data) has better performance than that of Method One using the single sEMG input, the estimation performance is still affected by inter-load (since the testing data are from all loads). For Method Three, due to the zero classification error, the estimation condition is same as intra-load which has a substantially smaller mean RMSE than that of the inter-load. In comparison with Method One which collects only the sEMG data as the training set in multiple loads, there is a better estimation performance in Method Two and Method Three using sensor fusion technologies (sEMG and force sensors).

We also find that different speeds can affect the performance of elbow angle estimation. As shown in Table II, the total mean overall RMSE under V2 $\left(18.57^{\circ}\right)$ is smaller than that under V1 $\left(22.09^{\circ}\right)$ and $\mathrm{V} 3\left(20.65^{\circ}\right)$; as shown in Fig. 8, the mean RMSEs under V2 are smaller than that under V1 and V3 in Method One $\left(12.84^{\circ}\right)$ and Method Three $\left(8.16^{\circ}\right)$. Researchers have focused on the speed effect in the estimation performance. Shrirao [30] predicted the finger joint angle from the surface EMG measurements under three speeds (slow, medium and fast), and found that there was a highest RMSE under slow speed. Au [36] used EMG signals to evaluate the performance of shoulder and elbow motions estimation, and there was a $20^{\circ}$ difference between the maximum RMSE and minimum RMSE at different speeds and different accelerations. The possible reasons are: 1) the higher speed increases the collective firing of the underlying neurons and therefore leads to the increase of muscle activity and sEMG [30]. The functional state and the motion state of the nervous system are in an unstable region, which leads to a worse estimation performance. 2) The lower speed which is not constant during the motion period would affect the EMG amplitude. Because the speed cannot keep constant in a long motion period, the muscle force of subjects cannot maintain at an isometric level, which leads to a worse estimation performance either [37].

Fatigue is unsuitable in practice, and it has been proven that the EMG signal is affected by fatigue [38]. In this study, this was addressed by the specified rest between trials, and ensuring fatigue free data during one trial using subjective and objective tests. Although fatigue effect is outside the scope of this study, we suggest that the fatigue test should be required.

The future work will cover the following three aspects. First, the present results show that the proposed methods are applicable to joint angle estimation in able-bodied subjects. However, almost all users of exoskeletons and prostheses have neurological injury or disabled status. Thus, we should apply the proposed methods to the users who need to use exoskeletons to support their daily activities to validate the results. Second, subjects performed the flexion-extension movement without the exoskeletons and prostheses. However, once the exoskeleton or prosthesis is fitted on the arm, the recorded sEMG includes both arm and exoskeleton movement, thus the extra force is required to move the exoskeleton [39]. Third, the loads vary in a large range in practical use, which means a load may be not in the training range. Possible solutions include expansion of the training pool and application of autonomic learning algorithms. Further research on practical use will be carried out in our future work. 


\section{CONCLUSION}

In this paper, we have demonstrated that the load variation could have a substantial impact on the performance of elbow angle estimation. In order to resolve the effect, we have proposed three methods: 1) pooling training data from all loads together to form the pooled training data for the training model, 2) adding the measured load value (force sensor) as an additional input and 3) developing a two-step hybrid estimation approach based on load and sEMG. The proposed methods led to a significant reduction in the mean RMSE. It indicates that the proposed methods improved the performance and stability on joint angle estimation, and sensor fusion (sEMG sensor and force sensor) might be an efficient way to resolve the adverse effect of load variation. Our study represents a shift from the research domain (laboratory) towards the clinic domain (practice) by taking multiple loads into account.

\section{ACKNOWLEDGMENT}

The authors would like to extend their gratitude to Kejun Zhang, Chao Li and Hongxin Cao from College of Computer Science and Technology, Zhejiang University for assisting in the experimental process.

\section{REFERENCES}

[1] K. Kiguchi and Y. Hayashi, "An EMG-based control for an upper-limb power-assist exoskeleton robot," Syst. Man Cybern. Part B Cybern. IEEE Trans. On, vol. 42, no. 4, pp. 1064-1071, 2012.

[2] T. Lenzi, S. M. M. De Rossi, N. Vitiello, and M. C. Carrozza, "Intention-based EMG control for powered exoskeletons," Biomed. Eng. IEEE Trans. On, vol. 59, no. 8, pp. 2180-2190, 2012.

[3] F. Sylos-Labini, V. La Scaleia, A. d'Avella, I. Pisotta, F. Tamburella, G. Scivoletto, M. Molinari, S. Wang, L. Wang, E. van Asseldonk, and others, "EMG patterns during assisted walking in the exoskeleton," Front. Hum. Neurosci., vol. 8, p. 423, 2014.

[4] K. Kiguchi, T. Tanaka, and T. Fukuda, "Neuro-fuzzy control of a robotic exoskeleton with EMG signals," Fuzzy Syst. IEEE Trans. On, vol. 12, no. 4, pp. 481-490, 2004.

[5] S. Bitzer and P. van der Smagt, "Learning EMG control of a robotic hand: towards active prostheses," in Robotics and Automation, 2006. ICRA 2006. Proceedings 2006 IEEE International Conference on, 2006, pp. 2819-2823.

[6] F. H. Chan, Y.-S. Yang, F. K. Lam, Y.-T. Zhang, and P. A. Parker, "Fuzzy EMG classification for prosthesis control," Rehabil. Eng. IEEE Trans. On, vol. 8, no. 3, pp. 305-311, 2000.

[7] J. Wang, O. A. Kannape, and H. M. Herr, "Proportional EMG control of ankle plantar flexion in a powered transtibial prosthesis," in IEEE International conference on rehabilitation robotics (ICORR), Seattle, USA, 2013.

[8] T. Lorrain, N. Jiang, and D. Farina, "Influence of the training set on the accuracy of surface EMG classification in dynamic contractions for the control of multifunction prostheses," $J$. Neuroengineering Rehabil., vol. 8, no. 1, p. 25, 2011.

[9] H. Su, Z. Li, G. Li, and C. Yang, "EMG-Based neural network control of an upper-limb power-assist exoskeleton robot," in Advances in Neural Networks-ISNN 2013, Springer, 2013, pp. 204-211.
[10] Y. M. Aung and A. Al-Jumaily, "Estimation of upper limb joint angle using surface EMG signal," Int. J. Adv. Robot. Syst., vol. $10,2013$.

[11] T. Kawase, T. Sakurada, Y. Koike, and K. Kansaku, "Estimating joint angles from biological signals for multi-joint exoskeletons," in Systems, Man and Cybernetics (SMC), 2014 IEEE International Conference on, 2014, pp. 1470-1474.

[12] A. L. Delis, J. L. Carvalho, A. F. Da Rocha, R. U. Ferreira, S. S. Rodrigues, and G. A. Borges, "Estimation of the knee joint angle from surface electromyographic signals for active control of leg prostheses," Physiol. Meas., vol. 30, no. 9, p. 931, 2009.

[13] Y. M. Aung and A. Al-Jumaily, "sEMG based ANN for shoulder angle prediction," Procedia Eng., vol. 41, pp. 1009-1015, 2012.

[14] E. Scheme and K. Englehart, "Electromyogram pattern recognition for control of powered upper-limb prostheses: State of the art and challenges for clinical use," J. Rehabil. Res. Dev., vol. 48 , no. 6 , p. $643,2011$.

[15] N. Jiang, S. Dosen, K.-R. Müller, and D. Farina, "Myoelectric control of artificial limbs-Is there a need to change focus," IEEE Signal Process. Mag., vol. 29, no. 5, pp. 152-150, 2012.

[16] E. Scheme, A. Fougner, Ø. Stavdahl, A. D. C. Chan, and K. Englehart, "Examining the adverse effects of limb position on pattern recognition based myoelectric control," in Engineering in Medicine and Biology Society (EMBC), 2010, Annual International Conference of the IEEE, 2010, pp. 6337-6340.

[17] A. H. Al-Timemy, G. Bugmann, J. Escudero, and N. Outram, “A preliminary investigation of the effect of force variation for myoelectric control of hand prosthesis," in Engineering in Medicine and Biology Society (EMBC), 2013 35th Annual International Conference of the IEEE, 2013, pp. 5758-5761.

[18] X. Chen, X. Zhang, Z.-Y. Zhao, J.-H. Yang, V. Lantz, and K.-Q. Wang, "Hand gesture recognition research based on surface EMG sensors and 2D-accelerometers," in Wearable Computers, 2007 11th IEEE International Symposium on, 2007, pp. 11-14.

[19] X. Zhang, X. Chen, Y. Li, V. Lantz, K. Wang, and J. Yang, "A framework for hand gesture recognition based on accelerometer and EMG sensors," Syst. Man Cybern. Part Syst. Hum. IEEE Trans. On, vol. 41, no. 6, pp. 1064-1076, 2011.

[20] Z. Lu, X. Chen, Q. Li, X. Zhang, and P. Zhou, "A hand gesture recognition framework and wearable gesture-based interaction prototype for mobile devices," Hum.-Mach. Syst. IEEE Trans. On, vol. 44, no. 2, pp. 293-299, 2014.

[21] A. Fougner, E. Scheme, A. D. Chan, K. Englehart, and $\varnothing$. Stavdahl, "Resolving the limb position effect in myoelectric pattern recognition," Neural Syst. Rehabil. Eng. IEEE Trans. On, vol. 19, no. 6, pp. 644-651, 2011.

[22] S. H. Roy, Ms. Cheng, S.-S. Chang, J. Moore, G. De Luca, Sh. Nawab, and C. J. De Luca, "A combined sEMG and accelerometer system for monitoring functional activity in stroke," Neural Syst. Rehabil. Eng. IEEE Trans. On, vol. 17, no. 6, pp. 585-594, 2009.

[23] D. Novak, X. Omlin, R. Leins-Hess, and R. Riener, "Predicting targets of human reaching motions using different sensing technologies," Biomed. Eng. IEEE Trans. On, vol. 60, no. 9, pp. 2645-2654, 2013.

[24] R. Song and K. Y. Tong, "Using recurrent artificial neural network model to estimate voluntary elbow torque in dynamic situations," Med. Biol. Eng. Comput., vol. 43, no. 4, pp. 473-480, 2005.

[25] A. Luttmann, M. Jāger, J. Sökeland, and W. Laurig, "Electromyographical study on surgeons in urology. II. Determination of muscular fatigue," Ergonomics, vol. 39, no. 2, pp. 298-313, 1996.

[26] A. Smith and E. E. Brown, "Myoelectric control techniques for a rehabilitation robot," Appl. Bionics Biomech., vol. 8, no. 1, pp. 21-37, 2011. 
[27] C. J. De Luca, "Use of the surface EMG signal for performance evaluation of back muscles," Muscle Nerve, vol. 16, no. 2, pp. 210-216, 1993.

[28] C. J. De Luca, "The use of surface electromyography in biomechanics," J. Appl. Biomech., vol. 13, pp. 135-163, 1997.

[29] M. A. Oskoei and H. Hu, "Myoelectric control systems-A survey," Biomed. Signal Process. Control, vol. 2, no. 4, pp. 275294, 2007.

[30] N. A. Shrirao, N. P. Reddy, and D. R. Kosuri, "Neural network committees for finger joint angle estimation from surface EMG signals," Biomed. Eng. Online, vol. 8, no. 1, p. 2, 2009.

[31] S. Suryanarayanan, N. P. Reddy, and V. Gupta, "Artificial neural networks for estimation of joint angle from EMG signals," in Engineering in Medicine and Biology Society, 1995., IEEE 17th Annual Conference, 1995, vol. 1, pp. 823-824.

[32] Y. Koike and M. Kawato, "Estimation of dynamic joint torques and trajectory formation from surface electromyography signals using a neural network model," Biol. Cybern., vol. 73, no. 4, pp. 291-300, 1995.

[33] M.-F. Lucas, A. Gaufriau, S. Pascual, C. Doncarli, and D. Farina, "Multi-channel surface EMG classification using support vector machines and signal-based wavelet optimization," Biomed. Signal Process. Control, vol. 3, no. 2, pp. 169-174, 2008.

[34] C.-C. Chang and C.-J. Lin, "LIBSVM: a library for support vector machines," ACM Trans. Intell. Syst. Technol. TIST, vol. 2, no. 3, p. 27, 2011.

[35] O. Fujita, "Statistical estimation of the number of hidden units for feedforward neural networks," Neural Netw., vol. 11, no. 5, pp. 851-859, 1998.

[36] A. T. Au and R. F. Kirsch, "EMG-based prediction of shoulder and elbow kinematics in able-bodied and spinal cord injured individuals," Rehabil. Eng. IEEE Trans. On, vol. 8, no. 4, pp. 471-480, 2000.

[37] Z. Tang, K. Zhang, S. Sun, Z. Gao, L. Zhang, and Z. Yang, “An upper-limb power-assist exoskeleton using proportional myoelectric control," Sensors, vol. 14, no. 4, pp. 6677-6694, 2014.

[38] R. M. Enoka, S. Baudry, T. Rudroff, D. Farina, M. Klass, and J. Duchateau, "Unraveling the neurophysiology of muscle fatigue," J. Electromyogr. Kinesiol., vol. 21, no. 2, pp. 208-219, 2011.

[39] C. Cipriani, R. Sassu, M. Controzzi, and M. C. Carrozza, "Influence of the weight actions of the hand prosthesis on the performance of pattern recognition based myoelectric control: preliminary study," in Engineering in Medicine and Biology Society, EMBC, 2011 Annual International Conference of the IEEE, 2011, pp. 1620-1623.

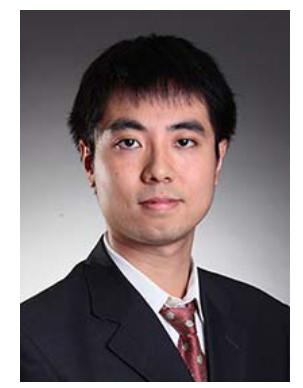

Zhichuan Tang (S'14) received the $\mathrm{Ph} . \mathrm{D}$. degree at the College of Computer Science, Zhejiang University, Hangzhou, China, in 2014. He was an Erusmus Mundus Scholar under cLINK Program from 2014 to 2015 , and is currently a postdoc research fellow with Faculty of Science and Technology, Bournemouth University, Poole, UK. His research interests include applied ergonomics, advanced exoskeleton control, signal processing of electromyography, human-computer interaction and industrial design.

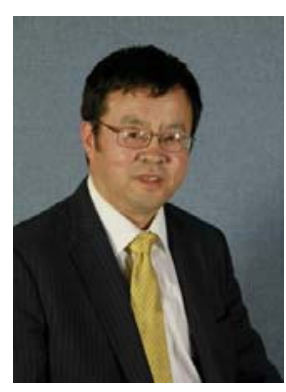

Hongnian Yu has the research interests in robotics, wireless networked control systems, RFID and its applications, mobile computing, modeling, scheduling, planning, and simulations of large discrete event dynamic systems with applications to manufacturing systems, supply chains, transportation networks and computer networks. He has published more than 200 research papers. He has held several research grants worth about three million pounds from EPSRC, the Royal Society, and the EU, AWM, as well as from industry. Prof. Yu is TC co-chair for the technical committee on Environmental Sensing, Networking and Decision Making within IEEE Systems, Man and Cybernetics.

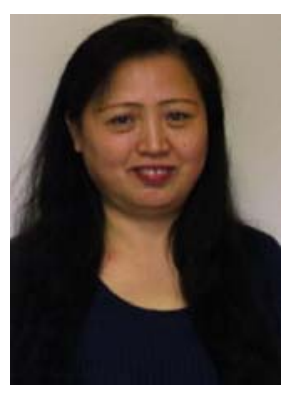

Shuang Cang is a Principal Academic Lecturer in the Faculty of Management, Bournemouth University, UK. She gained a $\mathrm{PhD}$ degree in Mathematics/Applied Mathematics. Then she worked in the Department of Computer Sciences at Exeter University and University of Wales (Aberystwyth). She spent over two years as Senior Statistician/Senior Analyst in the UK Government Research Laboratory and UK Government Department. She is currently managing three EU funded projects, Erasmus Mundus cLINK $(€ 2.5 \mathrm{M})$ and FUSION $(€ 3.05 \mathrm{M})$ projects, an EU Marie Curie IRSES RABOT project as the BU local coordinator. Her research interests include data mining, artificial intelligence, pattern recognition, multivariance statistics, forecasting, and segmentations. 